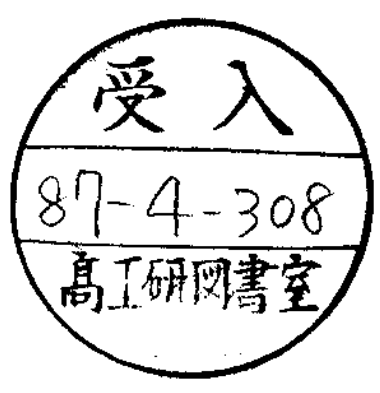

\title{
REDUCING THE RANK OF THE GAUGE GROUP IN ORBIFOLD COMPACTIFICATIONS OF THE HETEROTIC STRING
}

\author{
L.E. Ibàñez, H.P. Nilles and F. Quevedo
}

CERN-Geneva

\begin{abstract}
Using the fact that the space group of an orbifold is non-Abelian, we can reduce the rank of the four-dimensional gauge group obtained from orbifold compactification of the heterotic string. The embedding of the space group in the gauge degrees of freedom is performed by assigning shifts (Wilson lines) in the $\mathrm{E}_{8} \times \mathrm{E}_{8}$ lattice to the shifts in the six-dimensional lattice, and $\mathrm{E}_{8} \times \mathrm{E}_{8}$ Weyl rotations to the spatial discrete rotations. Reducing the rank of the gauge group is phenomenologically interesting for explaining the absence of extra low-energy gauge interactions.
\end{abstract}




\section{INTRODUCTION}

Constant background fields have played an important role in understanding toroidal compactifications of the hererotic string [1]. They have also enriched the class of models obtained from orbifold compactifications [2, 3]. In particular, the presence of Wilson lines can break the gauge group and control the number of families in orbifold models [3]. This mechanism has led to the explicit construction of phenomenologically interesting fourdimensional string models, with three families of chiral matter and $S U(3) \times S U(2) \times U(1)^{n}$ symmetries, based on the $Z$ orbifold [4].

Even though this is a very flexible way of constructing four-dimensional string models, it has the restriction of leaving the rank of the gauge group unchanged*). This is a consequence of the fact that the space group of the orbifold is embedded in an Abelian way in the gauge group. Large-rank gauge groups are also a characteristic feature of other constructions of chiral string models in four dimensions [6, 7]. For phenomenological reasons, it is desirable to reduce the rank of the gauge group in order that the absence of extra-low-energy gauge interactions may eventually be explained.

In this note we show how the rank of the gauge group can be reduced by considering non-Abelian embeddings of the space group $S$ in the gauge degrees of freedom. The Wilson lines are represented by shifts in the $\mathrm{E}_{8} \times \mathrm{E}_{8}$ lattice, whereas the embedding of the point group $P$ is made by Weyl rotations of the group lattice. More explicitly, the transformations defining the space group are

$$
x^{i} \longrightarrow(\theta x)^{i}+v^{i} \quad i=1, \ldots, 6
$$

where $\theta \in P$ is a discrete rotation and the shift $v^{i}$ is given by $v^{i}=n^{\alpha} e_{\alpha}^{i}$, with $e_{\alpha}$ being the lattice vectors and $n^{\alpha}$ integer coefficients. The embedding of the space group $s=\left\{\left(\theta, v^{i}\right)\right\}$ in the gauge degrees of freedom is defined by the transformations

*) The rank could be enlarged by considering background antisymmetric tensor fields and fixing the radius of the tori $[1,5]$. 


$$
X^{I} \longrightarrow(\oplus X)^{I}+v^{I}, \quad I=1, \ldots 16
$$

where $\Theta$ is an automorphism of the group lattice, i.e. an element of the Weyl group, and $v^{I}=n^{\alpha} a_{\alpha}^{I}$, where $a_{\alpha}^{I}$ are the wilson lines corresponding to the non-contractible loops defined by $e_{\alpha}$. The total discrete group of the orbifold is then $G=\left\{\left(\theta, v^{j} ; \Theta, v^{\mathbf{I}}\right\}\right.$; since, in general, the transformations $\left(\theta, v^{I}\right)$ do not commute, we can use this embedding to lower the rank of the gauge group. The homomorphism $\left(\theta, v^{i}\right) \rightarrow\left(\theta, v^{I}\right)$ has to be contrasted with previously considered embeddings where both $\theta$ and $v^{i}$ were in correspondence with shifts in the group lattice, i.e. $\left(\theta, v^{i}\right) \rightarrow\left(v^{I}, a^{I}\right)$. In the absence of Wilson lines, the two types of embeddings are equivalent [2], as we will see in the next section where all the non-equivalent embeddings of the $\mathrm{z}$ orbifold are reproduced in terms of Weyl rotations. Subsequently, we consider the presence of Wilson lines and show how the rank of the gauge group can be reduced in this formalism. A simple example in which $\mathrm{E}_{8}$ is broken to $\mathrm{SU}(3)^{3}$ is discussed in detail.

\section{WEYL ROTATIONS AND THE $z$ ORBIFOLD}

We want to make a correspondence between discrete spatial rotations and discrete rotations in the $E_{8} x_{8} E_{8}$ lattice. For the $\mathrm{z}$ orbifold, which is the $\mathrm{SU}(3)^{3}$ lattice modded out by $2 \pi / 3$ rotations, we want to embed the $\mathrm{SU}(3)^{3}$ rotations $\theta$ in the $\mathrm{E}_{8} \mathrm{x}$ $E_{8}$ lattice. Therefore we will consider the Weyl group of the SU(3) lattice; it is generated by reflections in the directions orthogonal to the roots of the group. If $e_{1}=(\sqrt{2}, 0)$ and $e_{2}=$ $(-1 / \sqrt{2}, \sqrt{3 / 2})$ represent the $\mathrm{SU}(3)$ simple roots, there are two independent reflections of the two-dimensional lattice vectors

$$
\Gamma_{i}(x)=x-\left(x, \rho_{i}\right) \rho_{i}, \quad i=1,2
$$

From $\Gamma_{i}$ we can construct the transformation

$$
\Theta \equiv \Gamma_{2}
$$

which rotates a two-dimensional vector by $2 \pi / 3$. We can now embed $\mathrm{SU}(3)^{3}$ (once or twice) in $\mathrm{E}_{8} \times \mathrm{E}_{8}$ and perform simultaneous $\theta$ rotations on each $\mathrm{SU}(3)$. There are four non-trivial, modularinvariant ways of doing the embedding: 
i) by associating the spatial twists to simultaneous $2 \pi / 3$ rotations in an $\mathrm{SU}(3)^{3}$ inside the first $\mathrm{E}_{8}$;

ii) by having one rotated $\mathrm{SU}(3)$ in the first $\mathrm{E}_{8}$ and two in the second;

iii) by having three rotated $\mathrm{SU}(3)$ 's in each $\mathrm{E}_{8}$;

iv) by having four rotated $S U(3)$ 's in the first $E_{8}$ and two in the second.

As we will see later, they correspond to the four independent ways of performing the embeddings by shifts in the $\mathrm{E}_{8} \quad \mathrm{x}_{8}$ lattice.

In order to have a better treatment of the SU(3)'s inside $E_{8} \times E_{8}$, we use an $\mathrm{SU}(3)^{4}$ basis in which an $E_{8}$ vector is written as

$$
\left(\frac{n_{2}-n_{3}}{\sqrt{2}}, \frac{2 n_{1}-n_{2}-n_{3}}{\sqrt{6}} ; \frac{n_{4}-n_{5}}{\sqrt{2}}, \frac{2 n_{6}-n_{4}-n_{5}}{\sqrt{6}} ; \frac{-\left(n_{7}+n_{8}\right)}{\sqrt{2}}, \frac{n}{\sqrt{6}} ; \frac{n_{7}-n_{6}}{\sqrt{2}}, \frac{n^{\prime}}{\sqrt{6}}\right)
$$
or

$$
\left(\frac{n_{2}-n_{3}}{\sqrt{2}}, \frac{2 n_{1}-n_{2}-n_{3}}{\sqrt{6}} ; \frac{n_{4}-n_{5}}{\sqrt{2}}, \frac{2 n_{6}-n_{4}-n_{5}}{\sqrt{6}} ; \frac{-\left(n_{7}+n_{8}+1\right)}{\sqrt{2}}, \frac{n_{1}+3}{\sqrt{6}} ; \frac{n_{7}-n_{8}}{\sqrt{2}}, \frac{n^{\prime}}{\sqrt{6}}\right)
$$

where $n=\Sigma_{i=1}^{6} n_{i}$ and $n^{\prime}=\Sigma_{i=1}^{3}\left(n_{i}-n_{i+3}\right)$, with the constraint $\Sigma_{i=1}^{8} n_{i}=$ even. In this basis, the $E_{8} \times E_{8}$ roots are expressed in the following way: the $24 \mathrm{SU}(3)^{4}$ roots plus 216 (= 8 x 27) from the set of roots

$$
D=\{ \pm(d ; d ; d ; 0), \pm(-d ; d ; 0 ;-d), \pm(d ; 0 ;-d ;-d), \pm(0 ;-d ; d ;-d)\}
$$

where $d$ represents any of the weights of each $s U(3): d_{1}=(1 / \sqrt{2}$, $1 / \sqrt{6}), \mathrm{d}_{2}=(-1 / \sqrt{2}, 1 / \sqrt{6})$, or $\mathrm{d}_{3}=(0,-2 / \sqrt{6})$.

We will describe as an example the canonical embedding of the $Z$ orbifold which, in the present formalism, corresponds to the embedding of the $\mathrm{SU}(3)^{3}$ rotations in the first $\mathrm{E}_{8}$. The gauge fields are obtained from the left-moving states which are invariant under the simultaneous $\theta$ rotations of three of the SU(3)'s inside $E_{8}$ (tensor the invariant right-moving states). From the eight oscillators which generate the $\mathrm{E}_{8}$ Cartan subalgebra in the heterotic string, only two are left invariant, corresponding to the $\mathrm{SU}(3)$ that is not rotated. The six roots of this $\mathrm{SU}(3)$ are also invariant, but all the other roots are rotated by $\Theta$ and 
therefore are not. However, invariant states appear from combinations of the type

$$
|P\rangle+|\Theta P\rangle+\left|\Theta^{2} P\right\rangle
$$

There are two of these invariant combinations coming from the roots of every rotated $\mathrm{SU}(3)$, and $72(=9 \times 8)$ from $\mathrm{D}$. Putting all the invariant states together, we obtain the gauge symmetry $E_{6} \times S U(3)$ from the first $E_{8}$; the second $E_{8}$ is left untouched. Note that since six oscillators are projected out, the role of the Cartan subalgebra has to be played by other states, for instance the six invariant combinations of the $\mathrm{SU}(3)^{3}$ roots.

The matter in the untwisted sector can be found in a similar way. The invariant combinations are now of the form $|p\rangle+\alpha|\Theta p\rangle$ $+\alpha^{2}\left|\Theta^{2} p\right\rangle$ or $|p\rangle+\alpha^{2}|\Theta p\rangle+\alpha\left|\theta^{2} p\right\rangle$ with $\alpha=e^{2 \pi i / 3}$. We obtain three copies of chiral matter transforming as $(3,27)$ of $\mathrm{SU}(3)$ $\mathrm{x}_{6}$ plus their antiparticles, coinciding with the results obtained from different formulations [2].

Let us now consider the twisted sector. The boundary conditions for the coordinates in this sector are

$$
\begin{array}{ll}
X^{A}(r)=[\Theta X(0)]^{A}+\sum m_{i} \rho_{i}^{A}, & A=1, \ldots 6 \\
X^{B}(r)=X^{B}(0)+\sum m_{i} \rho_{i}^{B}, & B=7,8
\end{array}
$$

where $e_{i}$ are the $E_{8} \times E_{8}$ basis vectors and $m_{i}$ are integers. The mode expansions of the $\mathrm{x}^{\mathrm{I}}$ 's are then

$$
\begin{aligned}
& X^{A}(\sigma, \tau)=X_{f}^{A}+\frac{i}{2} \sum \alpha_{n-Y_{3}}^{A} e^{-2 i\left(n-V_{3}\right)(\tau+\sigma)} \\
& X^{B}(\sigma, \tau)=X_{f}^{B}+\frac{1}{\pi} P^{B}(\tau+\sigma)+\frac{i}{2} \sum \alpha_{n}^{B} e^{-2 i n(\tau+\sigma)}
\end{aligned}
$$

where the fixed coordinates and winding vectors are

$$
\begin{aligned}
& X_{f}^{A}=\left[(\mathbb{I}-\Theta)^{-1} \sum m_{i} \rho_{i}\right]^{A} \\
& P^{B}=\sum m_{i} \rho_{i}^{B}
\end{aligned}
$$

where $p^{A}=0$ and $x_{f}^{B}$ is arbitrary. The number of independent 
fixed points (tori) found from (13) is nine but, as has been proved in Refs [2], [7], and [8], the effective degeneracy factor when we have only left-moving modes is the square root of the number of fixed points, which in this case is three. This can be explicitly seen by comparing the partition function with the one found from the embedding by shifts. The mass formula in the twisted sector is

$$
\frac{1}{4} m_{2}^{2}=\frac{1}{2} p^{2}+N_{L}-1 / 3
$$

where the zero point energy has the contribution from both the space-time and the internal oscillators. This fact can be used to see explicitly that we have to embed the whole $\mathrm{SU}(3)^{3}$ once or twice in $\mathrm{E}_{8} \times \mathrm{E}_{8}$, otherwise it would be impossible to have leftright level matching. From Eq. (14) we can see that the winding vector $\mathrm{p}^{\mathrm{I}}$ in $\mathrm{Eq} .(15)$ is an $\mathrm{E}_{8}$ lattice vector projected into the subspace that is left invariant under the action of $\theta, i . e . p=$ $\left[0,0 ; 0,0 ; 0,0 ;\left(n_{7}-n_{8}\right) / \sqrt{2}, n^{\prime} / \sqrt{6}\right]$, where we used the representation (5). This truncated lattice corresponds to an SU(3) weight lattice.

The ground state is a $(\overline{3}, 1)$ of $\mathrm{SU}(3) \times \mathrm{E}_{6}$, corresponding to the degeneracy factor $\sqrt{9}$. Massless states are obtained from $p^{2}$ $=0, \mathrm{~N}_{\mathrm{L}}=1 / 3$; exciting the ground state with the three spacetime oscillators we get $3(\overline{3}, 1)$ of $\mathrm{SU}(3) \mathrm{x} \mathrm{E}_{6}$, and exciting it with the internal oscillators we obtain 9 states. They combine with the 18 states obtained from the $6 \mathrm{sU}(3)$ weights with length $\mathrm{p}^{2}=2 / 3\left(\mathrm{~N}_{\mathrm{L}}=0\right)$, times the degeneracy factor $\sqrt{9}$, to form a $(1,27)$ of $\mathrm{SU}(3) \times \mathrm{E}_{6}$. Taking into account the extra degeneracy factor, coming from the 27 fixed points of the six-dimensional lattice, we obtain $81(\overline{3}, 1)+27(1,27)$ from the twisted sector.

A similar analysis can be made for each of the other three consistent embeddings mentioned in section 2. The embedding ( $i i)$ leads to the symmetry $E_{7} \times U(1) \times S O(14) \times U(1)$, embedding (iii) to $\left[E_{6} \times S U(3)\right]^{2}$, and (iv) to $S U(9) \times S O(14) \times U(1)$. The matter content of each of these models also coincides with the one found in Ref. [3]. 


\section{REDUCING THE RANK OF THE GAUGE GROUP}

In the previous section we discussed twistings of the type $G=\left(\theta, v^{i} ; \theta, 0\right)$ in which one realizes a homomorphism $\theta \rightarrow \Theta$ in the orbifold. In this way we can reproduce the known results corresponding to the embedding through shifts $v^{I}$ in the $E_{8} x_{8}$ lattice $\theta \rightarrow v^{I}$. As we remarked in the Introduction, more general twistings can be obtained by considering the embedding of the whole space group $S$ into the gauge degrees of freedom. To a twist realized by Weyl rotations $(\theta, 0 ; \theta, 0)$, we can add Wilson lines $\left(1, \mathrm{v}^{i} ; 1, \mathrm{a}^{\mathrm{I}}\right)$. These two group-elements do not, in general, commute (in particular $\Theta \mathrm{a}^{\mathrm{I}} \neq \mathrm{a}^{\mathrm{I}}$ ) and this may lead to a reduction of the rank of the gauge group.

When we are in the presence of a Wilson line we can proceed as in Ref. [3]. In the untwisted sector of the orbifold, we have to project on wilson-line singlets. In particular, only the winding states (or combinations of them) which verify all the conditions

$$
\left((\Delta)^{n} a\right)^{I} P^{I}=\text { integer }, \quad n=1,2, \ldots m
$$

(where $\mathrm{m}$ is the order of the twist) survive as massless states. This projection usually lowers the rank since, as we remarked in the previous section, when we twist by weyl rotations some of the Cartan generators come from invariant combinations of winding states.

Concerning the twisted sectors of the orbifold, some of them feel the presence of the wilson lines and others do not [3]. For the ones which do not feel them (called 'trivially twisted' sectors in Ref. [3]), things work as described in the previous section. For the rest of the twisted sectors, one proceeds in an analogous way by looking for fixed points under the combined action of Weyl-rotation + shift. In this case there are three possibilities:

i) all the components of the shift $\mathrm{a}^{\mathrm{I}}$ are rotated by $\Theta$;

ii) none of the components of $a^{I}$ are rotated by $\theta$;

iii) some of the components of $a^{I}$ are rotated by $\theta$.

In the first case the number of fixed points under the combined action of Weyl-rotation + shift is the same as in the 
case without Wilson lines. Their coordinates are given by

$$
X_{f}^{A}=\left[(1-\Theta)^{-1} \sum m_{i}^{5} \rho_{i}+(1-\Theta)^{-1} a\right]^{A}
$$

where $\Sigma \mathrm{m}_{i}^{f} \mathrm{e}_{i}^{A}$ gives the $\mathrm{E}_{8} \times \mathrm{E}_{8}$ lattice shifts required to come back to the fixed points after a $\Theta$ rotation. The last term, which is due to the presence of the wilson lines, does not change the number of fixed points. Thus the degeneracy of the ground state of the twisted sector does not change with the addition of this type of Wilson line. Notice that in case (i) there is no restriction on the length of the shift $a^{I}$. This is to be contrasted with the case in which one realizes both the twist and the wilson lines through shifts. In the latter case, if $\theta$ rotates all $v^{i}$, it is necessary $[2,3]$ for $m\left(v^{I}+a^{I}\right)$ to be in the $E_{8} \times E_{8}$ lattice, where $m$ is the order of the twisting and $\mathrm{v}^{\mathrm{I}}\left(\mathrm{a}^{\mathrm{I}}\right)$ denote the twist (Wilson line) acting on the gauge degrees of freedom.

The addition of a shift $a^{I}$ which is completely rotated by $\theta$ does not modify the form of the twisted-sector mass operator. It does not shift any momenta since the twisting by $\Theta$ forbids winding states. Also, the oscillator states, the degeneracy of the twisted vacua, and the zero-point energies are unmodified. Thus there will be level matching between the left- and right-movers if this matching were there before adding the wilson line. So one expects a modular-invariant result. This is also reasonable since the shift is not required to be of finite order. The same happens in the simple case of Wilson lines on top of an untwisted torus. No extra modular invariance constraints appear for this case.

In case (ii) above, $\theta a^{I}=a^{I}$; hence our group of twistings inside $\mathrm{E}_{8} \times \mathrm{E}_{8}$ is Abelian. This is equivalent to the case considered in Ref. [3] in which both the twists and the wilson lines are represented through shifts in the lattice. Finally, in case (iii), in which some of the components of $a^{I}$ are rotated by $\theta$ (but not all), the situation is more complicated. In this case the shift $\mathrm{a}^{I}$ will add up to the winding momenta, and level matchching is no longer guaranteed. However, one may add some extra pieces to the shift (e.g. in the other $\mathrm{E}_{8}$ ) in order to restore modular invariance. For the purpose of illustrating how we can 
easily lower the rank of the gauge group, we will concentrate on the simplest non-trivial case, in which the shift $a^{I}$ is completely rotated by $\Theta$.

\section{EXAMPLES}

We now describe a simple example in which one of the $\mathrm{E}_{8}$ 's is broken down to $\mathrm{SU}(3)^{3}$. Let us consider the $\mathrm{z}$ orbifold with an embedding which associates four $2 \pi / 3$ rotations in the $\mathrm{SU}(3)^{4}$ lattice inside the first $E_{8}$, and two $2 \pi / 3$ rotations in an $S U(3)^{2}$ subgroup of the second $E_{8}$. It is easy to check that the gauge group is broken to $\mathrm{SU}(9) \times \mathrm{SO}(14)^{\prime} \mathrm{x} U(1)^{\prime}$. For example, the 80 generators of $\mathrm{SU}(9)$ are obtained as follows. Eight generators (the Cartan subalgebra) come from the $2 \times 4=8$ combinations of $\mathrm{SU}(3)^{4}$ root states which are left invariant under the simultaneous rotation in the four $\mathrm{SU}(3)$ 's roots. The other 72 generators come from the $9(4+4)=72$ combinations of root-states in $\mathrm{D}$ [Eq. (7)] which are left invariant under the same operation. There are no massless states associated with the oscillators since the eight of them are modded. Thus we have an SU(9) gauge symmetry left unbroken from the first $E_{8}$. There is also chiral matter coming from the untwisted sector. This corresponds to states which get a factor $\alpha=\exp i(2 \pi) / 3$ upon the action of the fourfold rotation. There are 84 such states: 4 oscillator modes (the other 4 go as $\alpha^{2}$ ); 8 linear combinations of $\mathrm{SU}(3)^{4}$ root states [combinations of the form $\left.\left|p^{I}\right\rangle+\alpha^{2}\left|\theta p^{I}\right\rangle+\alpha\left|\theta^{2} p^{I}\right\rangle\right]$; and again $9(4+4)=72$ linear combinations of root states of the ' $D$ ' type. Altogether they fill an 84 representation (three-index totally antisymmetric) of $S U(9)$. There are three from the triple degeneracy of the right-movers. One can check in the same way that there are also three multiplets transforming as $\left(\underline{1}, \underline{14}{ }^{\prime}\right)+$ (1, 64') with respect to the rest of the gauge group $S O(14)^{\prime} \mathrm{x}$ $\mathrm{U}(1)$ '.

Concerning the twisted sector, one can check that there are 81 fixed points which are left invariant under the action of the complete weyl rotation (other would-be fixed points can be shown to be equivalent by an $\mathrm{E}_{8}$ lattice translation to those 81). since this refers to purely left-moving coordinates, the actual degeneracy of the vacuum will be $9=\sqrt{81}$. Since in the SU(9) sector all coordinates are modded, there are no winding states 
and the whole twisted spectrum is obtained by acting with oscillators on the ninefold vacuum. In fact in this case the zeropoint energy also vanishes and the twisted mass operator is symply given by the oscillator number $\tilde{\mathrm{N}}$. Thus the vacuum state $(\tilde{N}=0)$ is massless and, in fact, corresponds to a $\underline{\bar{g}}$ under $\mathrm{SU}(9)$. Since in the $\mathrm{Z}$ orbifold there are 27 fixed points with respect to the space group, we have altogether 27 g''s from the twisted sector. These precisely cancel the SU(9) anomaly given by the untwisted matter ( $3 \underline{84}$ ).

Up to now we have just reproduced one of the non-standard $z$ orbifold embeddings in terms of weyl rotations. Let us now add to this a Wilson line. This was described in detail for the Abelian embedding case in Ref. [3]. It is equivalent to a homomorphism $e_{\alpha} \rightarrow a_{\alpha}^{I}(\alpha=1, \ldots, 6)$ in which we associate a shift in the $E_{8} \times E_{8}$ lattice with any of the six non-trivial directions of the space-group torus. Since $\theta e_{\alpha}=e_{\alpha+1}(\alpha=1,3,5)$ in the SU( 3$)^{3}$ orbifold lattice, only three of the wilson lines are independent, and we have

$$
\left(\theta a_{\alpha}\right)^{I}=a_{\alpha+1}^{I} \quad, \quad \alpha=1,3,5
$$

Let us take as an example a single Wilson line $a_{1}$ around the direction $e_{1}^{i}$ corresponding to the vector $(\sqrt{2}, 0)$ in the first of the three SU(3) lattices of the $z$ orbifold. There is also necessarily a Wilson line $a_{2}^{I}=\left(\theta a_{1}\right)^{I}$ around the $e_{2}=\theta e_{1}$ direction. To be more specific, consider the shifts

$$
\begin{aligned}
& a_{1}^{I}=\lambda\left(d_{1} ; 0 ; 0 ; 0\right) \\
& a_{2}^{I}=\left(\Theta a_{1}\right)^{I}=\lambda\left(d_{2} ; 0 ; 0 ; 0\right)
\end{aligned}
$$

where $\lambda$ is a rescaling parameter and the $d_{i}$ are the $S U(3)$ weight vectors already introduced in section 2 . We have used an explicit $\mathrm{SU}(3)^{4}$ notation. Notice that these shifts are completely rotated by $\theta$ so that they are of the type (i) described in section 3. Notice also that the present embedding is in general non-Abelian. For example, the operations $(\theta, 0 ; \theta, 0)$ and $\left(1, e_{1} ;\right.$ $1, a_{1}^{I}$ ) do not commute. However, in the present example, for $\lambda=1, a_{1}^{I}$ and $a_{2}^{I}$ are equivalent through an SU(3) lattice element. Thus for $\lambda=1$ the embedding is Abelian and the rank 
cannot be lowered. For general shift length ( $\lambda \neq$ integer) this is not the case. We must recall that for shifts completely rotated by $\Theta$, the length is not constrained.

Let us start now with the untwisted sector. Of the $80 \mathrm{SU}(9)$ generators, only the combinations of winding states verifying $\mathrm{p}^{\mathrm{I}} \cdot \mathrm{a}_{1}^{\mathrm{I}}=$ integer, $\mathrm{p}^{\mathrm{I}} \cdot \mathrm{a}_{2}^{\mathrm{I}}=$ integer will survive. For arbitrary $\lambda$ there are only 24 states satisfying this. There are $2 \times 3=6$ linear combinations of the $\mathrm{SU}(3)^{3}$ root states corresponding to the three SU(3)'s left untouched by the shifts in Eqs. (19). There are also $9+9=18$ combinations of states of the type $\pm\left(0 ;-d_{i} ; d_{j} ;-d_{k}\right)$. One can check that, as might have been guessed, these 24 states correspond to the generators of an $\mathrm{SU}(3)^{3}$ group. The rank of the initial gauge group $\mathrm{E}_{8}$ has been lowered to 6 . In fact for $\lambda=1$ the gauge symmetry is enlarged since there are two further states surviving the projection. These are the two linear combinations of root states of the first $S U(3)$ (the one 'touched' by the shift). Notice that for $\lambda=1, \mathrm{a}_{1}^{\mathrm{I}}$ and $\mathrm{a}_{2}^{\mathrm{I}}$ are just $\mathrm{sU}(3)$ weights having an integer scalar product with root vectors. Thus for $\lambda=1$, the gauge group is $\mathrm{SU}(3)^{3} \mathrm{xU}(1)^{2}$ and the rank is not lowered.

There is also chiral matter in the untwisted sector which survives the projection. Of the 84 states existing before adding the wilson line, only 28 survive. These are: 4 modded oscillator states; $3 \times 2=6$ linear combinations of the last three $\mathrm{SU}(3)$ root states; and $(9+9)=18$ combinations of states of the form $\pm\left(0 ;-d_{j} ; d_{j} ;-d_{k}\right)$ (the combinations are of the type $|p\rangle+\alpha^{2}|\theta p\rangle$ $\left.+\alpha\left|\Theta^{2} \mathrm{p}\right\rangle\right)$. One can show that under $\mathrm{SU}(3)^{3}$ these chiral fields have the quantum numbers

$$
28=(3,3,3)+(1,1,1)
$$

Again there is a factor of 3 due to the triple degeneracy of the right-movers.

Concerning the twisted sector, it is not greatly changed by the presence of the Wilson lines. As was explained in detail in Ref. [3], the twenty-seven $z$-orbifold fixed points split into three sets of nine fixed points. The first nine ('trivially twisted') do not feel the presence of the wilson line since they correspond to the nine fixed points that have vanishing coordi- 
nates under the first two dimensions and thus do not have any associated gauge shift. These nine fixed points just have the usual nine $\underline{9}^{\prime} s$ of $\mathrm{SU}(9)$ [conveniently written in terms of $\mathrm{SU}(3)^{3}$ ]. The second set of nine fixed points have the bosonic coordinates' boundary conditions:

$$
\begin{aligned}
& X^{i}(r)=(\theta X(0))^{i}+e_{1}^{i}+(\text { shifts without wilson lines) } \\
& X^{i}(r)=(\Theta X(0))^{I}+a_{1}^{I}
\end{aligned}
$$

In the gauge coordinates, as we remarked above, the number of fixed points under combined twist and shift is unchanged and still equal to 81 . Thus the degeneracy of the vacuum is also ninefold. Finally, the third set of nine fixed points have the bosonic coordinates' boundary conditions,

$$
\begin{aligned}
& X^{i}(r)=(\theta X(0))^{i}+e_{1}^{i}+e_{2}^{i}+(\text { shifts without Wilson lines) } \\
& X^{I}(r)=(\Theta X(0))^{I}+a_{1}^{I}+a_{2}^{I},
\end{aligned}
$$

and again we have a ninefold vacuum. Thus all three sectors have the same quantum numbers under $\mathrm{SU}(3)^{3}$ corresponding to

$$
|\overline{9}\rangle=(1,1, \overline{3})+(1, \overline{3}, 1)+(\overline{3}, 1,1)
$$

Putting all pieces together we get an $\mathrm{SU}(3)^{3}$ model with chiral matter:

Untwisted: $3[(3,3,3)+(1,1,1)]$,

twisted: $27[(1,1, \overline{3})+(1, \overline{3}, 1)+(\overline{3}, 1,1)]$

Notice how the $\mathrm{sU}(3)^{3}$ anomalies of the untwisted sector are cancelled in a non-trivial (chiral) way by the twisted sector. Thus we have a consistent model in which the rank of the gauge group has been lowered by two units.

As we mentioned above, for $\lambda=1$ the gauge group is enlarged to $\mathrm{SU}(3)^{3} \times \mathrm{U}(1)^{2}$. In this case the massless particle content is exactly as in the $\mathrm{SU}(3)^{3}$ except for the appearance of the couple of $U(1)$ supermultiplets plus $3 \times 2=6$ additional 
chiral singlets. Since this case $(\lambda=1)$ is Abelian it should be possible to obtain an equivalent $\mathrm{SU}(3)^{3} \times \mathrm{U}(1)^{2}$ model by realizing both the twist and the wilson lines through shifts (as in Ref. [3]). In fact, using the shift $v^{I}=(1 / 3,1 / 3,1 / 3,1 / 3,2 / 3$, $0, \ldots, 0)(2 / 3,0, \ldots, 0)$ and the Wilson line $a^{I}=(2 / 3,0,0$, $0,-1 / 3,1 / 3,0,0)(0, \ldots, 0)$ we recover the above results. These equivalent Abelian models are useful for checking that the quantum numbers obtained through weyl-rotations + shift are correct.

We can easily find other examples with reduced rank. If we start with a model with three weyl rotations inside one $\mathrm{E}_{8}$ (as in Section 2) and we add a shift analogous to the ones in Eq. (17), we obtain a model with gauge group $S O(8) \times U(1)^{2}$. We have chiral matter in the untwisted sector transforming as $3\left(\underline{8}_{s}+\underline{8}_{v}+\underline{8}_{a^{\prime}} 1\right)$ and chiral fields from the twisted sector as $27\left(\underline{8}_{\mathrm{s}}+\underline{8}_{\mathrm{v}}+\underline{8}_{\mathrm{a}}+\right.$ $3 \underline{1})+243 \underline{1}$. Using the same type of Wilson line for the case of two Weyl rotations inside one $E_{8}$, we get a gauge group $S U(6) \mathbf{x}$ $U(1)$. In this case the untwisted matter transforms as $3(20+2 \underline{1})$ and the twisted matter depends on the number of rotations that are acting on the second $\mathrm{E}_{8}$, but in any case it is always nonchiral. Finally, the addition of a Wilson line, as in Eq. (19), in the case of only one weyl rotation inside the first of the $E_{8}$ 's will lead to an $E_{6}$ gauge group with chiral matter transforming as singlets. We remark that in all these cases we assume that the shift is completely rotated by $\theta$. Also, in all the above cases for $\lambda=1$, the gauge symmetry is enlarged and a couple of $U(1)$ 's appear along with six chiral matter singlets. For all these rank-8 $(\lambda=1)$ models, we can find equivalent ones with both the twisting and the wilson lines realized through shifts.

The rank can be lowered by more than two units. For instance, consider a wilson line shift $a_{1}^{I}=\lambda\left(d_{1} ; d_{1} ; 0 ; 0\right)$ in the $\mathrm{E}_{6} \mathrm{x} \mathrm{SU}(3)$ example discussed in the previous sections. It can easily be seen that the symmetry is broken down to $S U(4) \times U(1)$ and the rank is lowered by four units. The possibilities are many (we may also add a second or a third wilson line) and the method of lowering the rank is very efficient. We hope that all 
the above examples illustrate sufficiently how the mechanisms of simultaneous embedding of Weyl rotations and shifts work.

\section{CONCLUSIONS}

In this letter we have argued that the non-Abelian structure of the space group of orbifolds can be fully embedded inside the gauge degrees of freedom. This is done by assigning Wey 1 rotations to the spatial discrete rotations, and assigning shifts in the $\mathrm{E}_{8} \times \mathrm{E}_{8}$ lattice to the shifts in the six-dimensional lattice. This approach keeps a nice parallelism between the space group and the group of gauge twistings, which is more natural than the usual approach in the bosonic construction which associates shifts in $\mathrm{E}_{8} \mathrm{x}_{8}$ with both the twistings and the wilson lines. Furthermore, since the embedding is in general non-Abelian (although the point group may be Abelian), the rank of the gauge group may be lowered by having both the twistings and the wilson lines not commuting. The examples shown in the text were based on the $z$ orbifold and assumed that the wilson line shifts were completely rotated by the weyl generator $\theta$. However, the present approach is completely general and therefore applies to much more general situations. In particular, this kind of non-Abelian embeddings can be repeated for the case of left-right asymmetric orbifolds [7].

The interest in lowering the rank of the gauge group is not just academic. Toroidal and orbifold compactifications usually lead to very large gauge groups. Even though very often the interesting particles (quarks, leptons, etc.) do not couple to a large sector of the gauge group ('hidden sector'), the physical gauge group is usually still too large. For example, in Ref. [4] there are models with three generations of quarks and leptons and gauge group $S U(3) \times S U(2) \times U(1)^{n}$. Ideally, we would like to get rid of the extra $U(1)$ 's and obtain just the standard Model with the minimal particle content. We hope that the approach presented in this paper will help in finding realistic compactifications that are as close as possible to the standard Model. 


\section{REFERENCES}

[1] K.S. Narain, Phys. Lett. 169B (1986) 41.

K.S. Narain, M.H. Sarmadi and E. Witten, Nucl. Phys. B279 (1987) 369.

P. Ginsparg, Comment on toroidal compactification of heterotic superstrings, Harvard preprint HUTP-86/A053 (1986).

[2] L. Dixon, J. Harvey, C. Vafa and E. Witten, Nucl. Phys. B261 (1985) 678 and B274 (1986) 285.

[3] L.E. Ibañez, H.P. Nilles and F. Quevedo, Orbifolds and Wilson lines, preprint CERN-TH.4611/86 (1986).

[4] L.E. Ibânez, J.E. Kim, H.P. Nilles and F. Quevedo, Orbifold compactifications with three families of $\operatorname{SU}(3) \times \operatorname{SU}(2) \mathrm{x}$ $\mathrm{U}(1)^{\mathrm{n}}$, preprint CERN-TH.4661/87 (1987).

[5] M. Mueller and E. Witten, Twisting toroidally compactified heterotic strings with enlarged symmetry groups, Princeton preprint (1986).

[6] H. Kawai, D.C. Lewellen and S.-H.H. Tye, Construction of fermionic string models in four dimensions, Cornell preprint CLNS 86/751 (1986).

W. Lerche, D. Lüst and A.N. Schellekens, Chiral fourdimensional heterotic strings from self-dual lattices, preprint CERN-TH.4590/86 (1986).

I. Antoniadis, C. Bachas and C. Kounnas, Four-dimensional superstrings, Ecole polytechnique and Berkeley preprint A76.11286/LBL-22709 (1986).

[7] K.S. Narain, M.H. Sarmadi and C. Vafa, Asymmetric orbifolds, Harvard preprint HUTP-86/A089 (1987). 
[8] D. Altschüler, Ph. Bérana, J. Lacki and I. Roditi, string Models with twisted vertex operators, Univ. of Geneva preprint UGVA-DPT 1986/09-518 (1986). 